\title{
Signatures of Alfvén waves in the polar coronal holes as seen by EIS/Hinode ${ }^{\star}$
}

\author{
D. Banerjee ${ }^{1}$, D. Pérez-Suárez ${ }^{2}$, and J. G. Doyle ${ }^{2}$ \\ 1 Indian Institute of Astrophysics, Bangalore 560034, India \\ e-mail: dipu@iiap.res.in \\ 2 Armagh Observatory, College Hill, Armagh BT61 9DG, N. Ireland
}

Received 1 April 2009 / Accepted 10 June 2009

ABSTRACT

\begin{abstract}
Context. We diagnose the properties of the plume and interplume regions in a polar coronal hole and the role of waves in the acceleration of the solar wind.

Aims. We attempt to detect whether Alfvén waves are present in the polar coronal holes through variations in EUV line widths. Methods. Using spectral observations performed over a polar coronal hole region with the EIS spectrometer on Hinode, we study the variation in the line width and electron density as a function of height. We use the density sensitive line pairs of Fe XII $186.88 \AA$ and $195.119 \AA$ A and Fe XIII $203.82 \AA$ and $202.04 \AA$.

Results. For the polar region, the line width data show that the nonthermal line-of-sight velocity increases from $26 \mathrm{~km} \mathrm{~s}^{-1}$ at $10^{\prime \prime}$ above the limb to $42 \mathrm{~km} \mathrm{~s}^{-1}$ some $150^{\prime \prime}$ (i.e. $\sim 110000 \mathrm{~km}$ ) above the limb. The electron density shows a decrease from $3.3 \times 10^{9} \mathrm{~cm}^{-3}$ to $1.9 \times 10^{8} \mathrm{~cm}^{-3}$ over the same distance.

Conclusions. These results imply that the nonthermal velocity is inversely proportional to the quadratic root of the electron density, in excellent agreement with what is predicted for undamped radially propagating linear Alfvén waves. Our data provide signatures of Alfvén waves in the polar coronal hole regions, which could be important for the acceleration of the solar wind.
\end{abstract}

Key words. Sun: corona - Sun: oscillations - Sun: UV radiation - line: profiles - waves

\section{Introduction}

Over the past decade, data from Ulysses show the importance of the polar coronal holes, particularly at times near solar minimum, for the acceleration of the fast solar wind. Acceleration of the quasi-steady, high-speed solar wind requires additional energy in the supersonic region of the flow. It has been shown theoretically that Alfvén waves from the Sun can accelerate the solar wind to these high speeds. Until now this is the only mechanism shown to enhance the flow speed of the basically thermally driven wind to the high flow speeds observed in interplanetary space. The Alfvén speed in the corona is quite high, so Alfvén waves can carry a significant energy flux even for low wave energy density. Direct observations of Alfvén waves has gained momentum after the launch of the Hinode satellite. Recent reports of detections of low-frequency $(<5 \mathrm{mHz})$, propagating Alfvénic motions in the solar corona (Tomczyk et al. 2007, from coronagraphic observations) and chromosphere (De Pontieu et al. 2007b) and their relationship with chromospheric spicules observed at the solar limb (De Pontieu et al. 2007a) with the Solar Optical Telescope (SOT; Tsuneta et al. 2008b) on Hinode (Kosugi et al. 2007) have widened interest in the subject. If MHD waves play an important role in accelerating high-speed streams and coronal heating, then this should be observed via a broadening of spectral lines, increasing with radial distance in the inner corona.

There have been several off-limb spectral line observations to search for Alfvén wave signatures. Measurements of

* Table 1 and Fig. 6 are only available in electronic form at http://www . aanda. org ultraviolet $\mathrm{Mg}$ X line widths during a rocket flight showed an increase in width with height to a distance of $70000 \mathrm{~km}$ (Hassler et al. 1990). Also, coronal hole Fe X spectra taken at Sacramento Peak Observatory showed an increase in the line width with height (Hassler \& Moran 1994). SUMER/SoHO (Wilhelm et al. 1995) were used to record the off-limb, height-resolved spectra of an Si VIII density sensitive line pair, in equatorial coronal regions (Doyle et al. 1998; Wilhelm et al. 2005) and in polar coronal holes (Banerjee et al. 1998; Wilhelm et al. 2004). The measured variation in line width with density and height supports undamped wave propagation in coronal structures. This was strong evidence of outwardly propagating undamped Alfvén waves in the corona, which may contribute to coronal heating and high-speed solar wind in the case of coronal holes. We revisit the subject with the new EIS instrument on Hinode and compare with our previous results as recorded by SUMER/SoHO. In the present study, we also make a full scan in the off-limb polar region to study the differences between the plume and interplume regions and to address the question as to whether the plume or the interplume is the preferred channel for the acceleration of the wind. Analyses of SUMER (e.g. Banerjee et al. 2000; Teriaca et al. 2003) and UVCS (e.g. Giordano et al. 2000) data have shown that the width of UV lines in interplumes is greater than in plume regions, indicating that interplumes as the site where energy is preferentially deposited and, possibly, the fast wind emanates. In this letter we concentrate on the density diagnostic capability of the density-sensitive line pairs of Fe XII $186.88 \AA$ and $195.119 \AA$ (Young et al. 2009) and Fe XIII 203.82 $\AA$ and 202.04 $\AA$ (Watanabe et al. 2009) for the off-limb coronal hole region. From a study of the variation of 
line width of the strongest line within our spectrum, we try to find signatures of propagating Alfvén waves.

\section{Observation and data reduction}

EIS onboard Hinode observes high-resolution spectra in two wavelength bands $170-211 \AA$ and $246-292 \AA$ with a spectral resolution of $0.0223 \AA$ per pixel (Culhane et al. 2007). We observed the north polar coronal hole on and off the limb with the $2^{\prime \prime}$ slit on 10 October 2007 (see left panel of Fig. 6, available online only, for a context image). The raster was run for more than four hours with 101 exposures of $155 \mathrm{~s}$, covering an area of $201.7^{\prime \prime} \times 512^{\prime \prime}$. We used the study arm_raster_ar, which was designed with nine windows containing lines from Fe VIII, Fe X, Fe XI, Fe XII, Fe XIII, Fe XIV, Fe XV, He II, Mg VI, O V, $\mathrm{O}$ VI, Si VII, and Al IX. All data have been reduced and calibrated with the standard procedures as given in the SolarSoft $(\mathrm{SSW})^{1}$ library. These standard subroutines include dark current subtraction, cosmic ray removal, flat field, hot pixel correction, and absolute calibration. We use the density sensitive line pairs of Fe XII 186.88 $\AA$ and 195.119 $\AA$ (Young et al. 2009) and Fe XIII 203.82 $\AA$ and 202.04 $\AA$ (Watanabe et al. 2009) for density diagnostics and use the strongest line of Fe XII 195.119 $\AA$ for the line width study. These lines are formed in the temperature range of $T=1$ to $2 \mathrm{MK}$. One can see from the $\mathrm{CHIANTI}^{2}$ (Landi et al. 2006) atomic data base that these line pairs have a very good density sensitivity between $7.0 \leq \log N_{\mathrm{e}}\left(\mathrm{cm}^{-3}\right) \leq 10.0$.

The Fe XII $195.12 \AA$ was fitted using a double Gaussian (see Young et al. 2009), considering the two main transitions at 195.119 and $195.179 \AA$ (with a 5\% contribution on average to the stronger component). The two transitions for Fe XII at 186.854 and 186.887 were fitted with a single Gaussian at $186.88 \AA$. For Fe XIII, the new CHIANTI updated version was used, which incorporates new $\mathrm{Fe}$ ion models as described in Watanabe et al. (2009). To address the issue of weaker signalto-noise as we go off-limb, a variable binning in the radial direction was performed, where after $1010^{\prime \prime}$ we initially binned over 27 pixels, finishing by binning over 42 pixels far off-limb. For Fe XIII we also binned over 5 spectra in the X direction. The "grating tilt" reported by Young et al. (2009), which clearly affects line ratios, was corrected by assuming that the tilt is linear and using the value found by Young et al. (2009) after our own comparison.

\section{Results}

The spectral line profile of an optically thin coronal emission line results from the thermal broadening caused by the ion temperature $T_{i}$, as well as by small-scale unresolved nonthermal motions and/or unresolved flows. Assuming that the instrumental profile can be expressed as Gaussian, the expression for the full width half maximum $(F W H M)$ is given as

$F W H M=\left[W_{\text {inst }}^{2}+4 \ln 2\left(\frac{\lambda}{c}\right)^{2}\left(\frac{2 k T_{i}}{M_{i}}+\xi^{2}\right)\right]^{1 / 2}$

where $T_{i}, M_{i}$, and $\xi$ are respectively the ion temperature, ion mass, and nonthermal velocity, while $W_{\text {inst }}$ is the instrumental width (for the $2^{\prime \prime}$ slit we use $62 \mathrm{~m}^{3}$ ), with the ion temperature

\footnotetext{
1 http: //www. lmsal. com/solarsoft/

2 http://www. solar.nrl.navy.mil/chianti.html

3 http://msslxr.mssl.ucl.ac.uk: 8080/eiswiki/Wiki.jsp? page $=2$ EISSlit
}

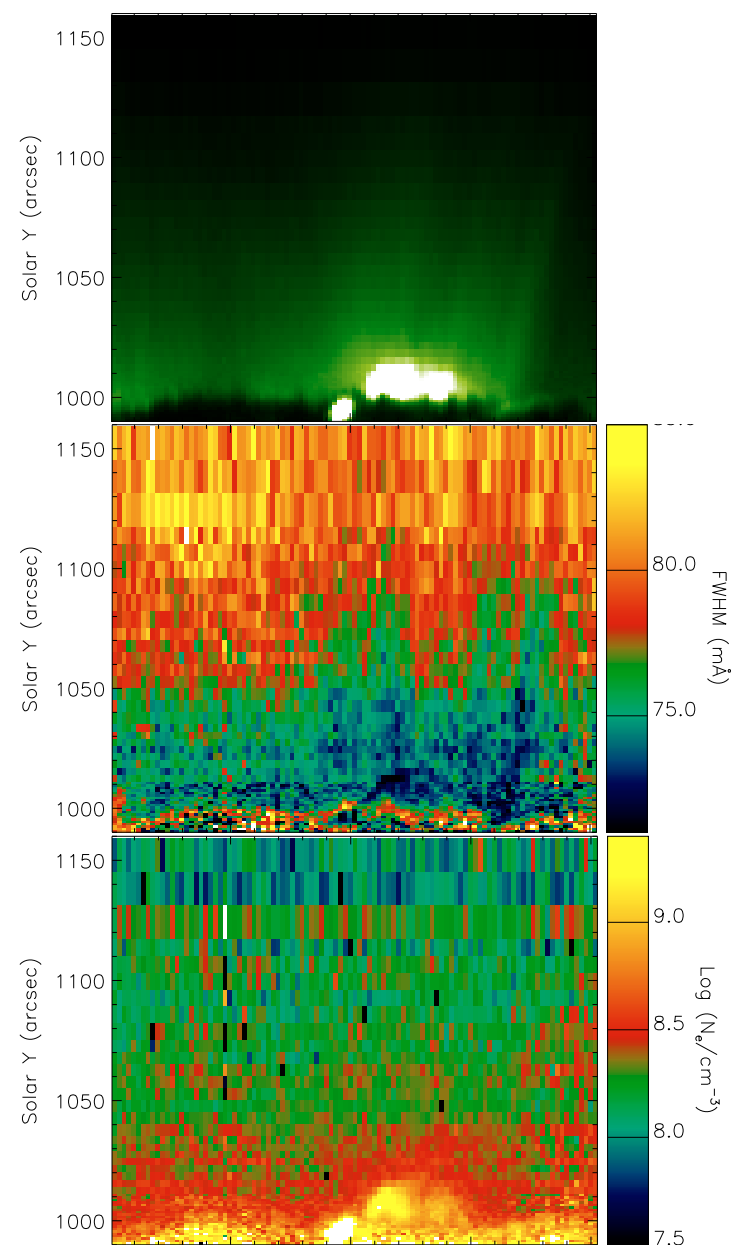

Fig. 1. Images of the north polar coronal hole off-limb region. From top to bottom: intensity, width, and density obtained from Fe XII lines.

assumed equal to the peak in the line formation temperature as given by CHIANTI. Figure 1 shows maps of line intensity and FWHM from Fe XII $195.12 \AA$ and electron density (from Fe XII) of the northern polar off-limb coronal hole region.

Observations have revealed that plasma conditions in polar plumes are quite different from interplume lanes (Hassler et al. 1997; Wilhelm et al. 1998). From these maps it is clear that plumes have slightly higher density and lower FWHM. Closer to the limb at the base of the plume (around $X=15$ ) one also finds the presence of a coronal bright point and it does affect the density values close to the limb. To look for a possible correlation/anti-correlation between the line intensity and the most probable speed, we concentrate on specific heights. As we go off the limb, Fig. 2 shows the line intensity and the FWHM along three strips tangent to the limb at different heights. We find evidence of an anti-correlation between the intensity and $F W H M$. If we go out farther away from the limb, the anticorrelation is weaker, because at these heights above the limb the plume structures have expanded slightly nonradially (see Fig. 1). This observed anti-correlation between intensity and width in polar plumes have been reported by Antonucci et al. (1997) and Noci et al. (1997) with the UVCS instrument and also by Hassler et al. (1997) and Banerjee et al. (2000) with SUMER. Now, to study the variations of different parameters namely density and $F W H M$ with height, we focus our attention on a fixed location, $X=15$, as a representative location for the plume and $X=-57$, as a representative case for the interplume. 


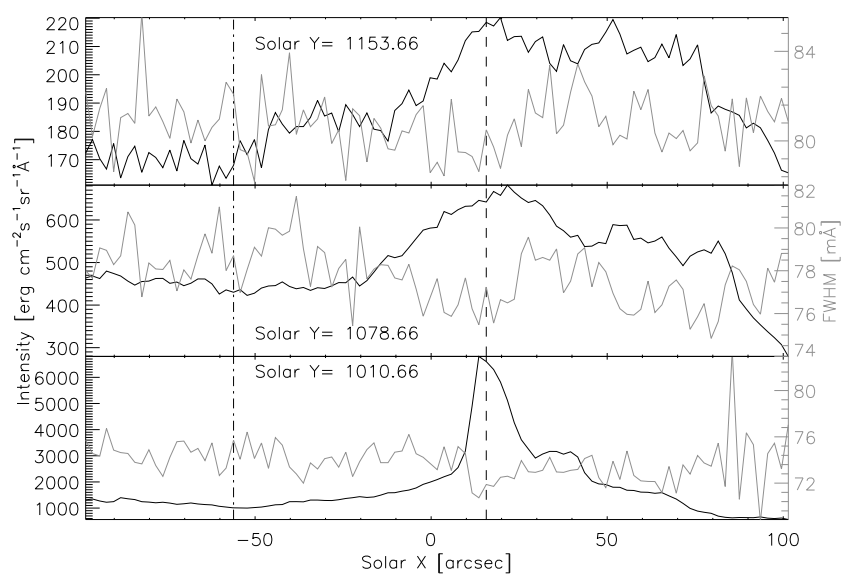

Fig. 2. A plot of the intensity (solid lines) and $F W H M$ (grey lines) as recorded by Fe XII $195 \AA$ along three strips tangent to the limb at different heights to illustrate the anti-correlation between intensity and velocity. The vertical lines show the position of the plume (dashed) and interplume (dot-dashed). The limb corresponds to $Y=1003$, as decided from the intensity peak of a low temperature line, Si VII.

In Fig. 3 we plot the variation of the density with height as recorded by Fe XIII (top panel) along plume and interplume (along the two dashed vertical lines as drawn in Fig. 2) ${ }^{4}$. The dashed lines in Fig. 3 correspond to a second order polynomial fit. One can clearly see that at the solar limb, the plume density is almost one order of magnitude higher than the interplume, but around $70^{\prime \prime}$ off-limb, the densities of the plume and interplume seem to be very close. The errors in deriving the electron density in Fig. 3 depends not only on the line strengths but also on the uncertainty within the atomic data, which can be upto $20 \%$ (Del Zanna \& Mason 2005). We should point out here that, for polar regions, these densities are higher than the previously published values. To validate our measurements, we also calculated the densities using Fe XII lines. For the same range of heights and along the same interplume lane, we compare the results in the middle panel of Fig. 3. It clearly shows that the densities are consistent (within their error bars) as recorded by two different line pairs of Fe XIII and Fe XII. In the lower panel of Fig. 3 we show a detailed comparison of our measured densities with earlier published values for the same range of heights for different coronal conditions (Wilhelm et al. 1998; van de Hulst 1950; Dolla \& Solomon 2008).

Our densities are marginally comparable to previously published values for plumes and for the quiet corona. This may stem form the new atomic data for Fe XIII and Fe XIII used in this analysis and/or contamination from the background quite sun. Assuming ionisation equilibrium and using Eq. (1), we now calculate the nonthermal speed and study its variation with height (see Fig. 4) for the Fe XII (as this is the strongest line and line widths are measured with better accuracies). The variation in the nonthermal velocity with height along the plume and interplume reveals that, consistently with height, the nonthermal velocity is slightly higher in the interplume than the plume. For comparison with previously reported results, we overplot the nonthermal velocity as derived from Si VIII $1445.75 \AA$ (Banerjee et al. 1998) as represented by triangles along with their best fit in Fig. 4 .

\footnotetext{
${ }_{4}$ A summary of the measured parameters and calculated values are tabulated in the on-line material, Table 1 for Fe XII and Fe XIII.
}

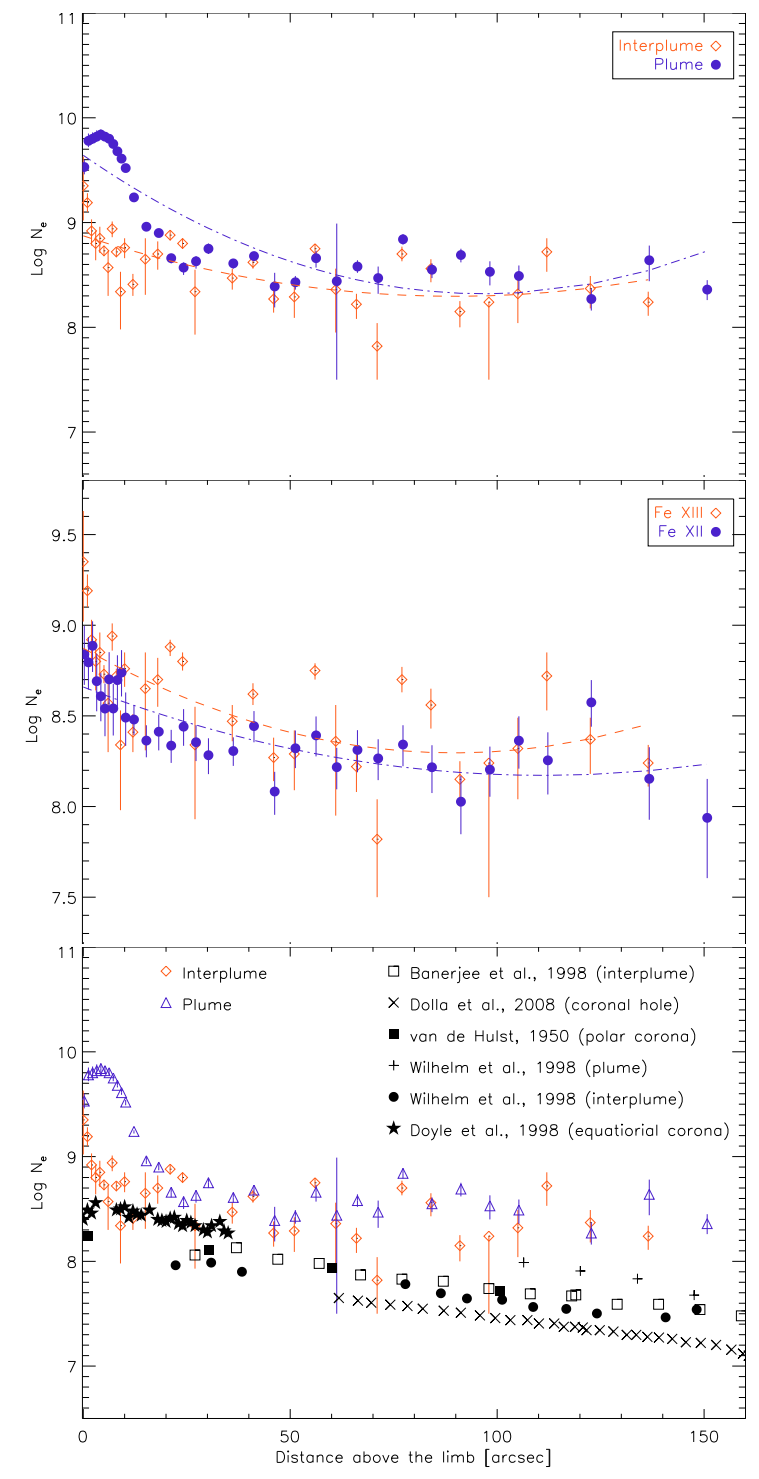

Fig. 3. Variation in density with height in the polar coronal hole region. The top panel shows results as recorded by Fe XIII, along plume and interplume regions. The middle panel shows the comparison of Fe XII and Fe XIII. The lower panel shows a comparison of density values as recoded by different instruments within the same radial distance.

\section{Discussion and conclusions}

Using the EIS spectrometer on Hinode, we have studied the variations in the line width and the electron density as a function of height. We used the density sensitive line pairs of Fe XII and Fe XIII, and calculated densities have comparable values (within their error bars). We must point out that the densities as reported here are consistently higher than previously published values (see Fig. 3). The difference may stem from real physical differences between the emitting regions of the coronal hole plasma around November 2007 and previous coronal conditions as recorded by other instruments (e.g. SUMER). Other factors could be the CHIANTI atomic models. There has been a recent revision in the Fe XIII atomic model (Watanabe et al. 2009), and we used the updated CHIANTI database for our calculation. We should also point out that, from the full scan image of the density (Fig. 6, available in the online version only), it shows that the off-limb coronal densities are close to the quiet Sun values and in fact somewhat higher than the on-disk coronal hole densities. 


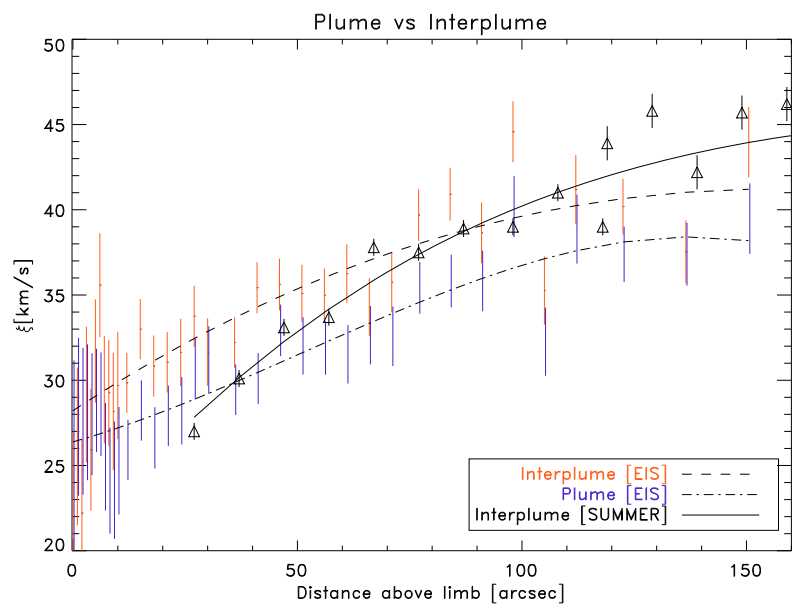

Fig. 4. Variation in nonthermal velocity with height as recorded by Fe XII $195 \AA$ along a polar plume and interplume (the present study). The solid line corresponds to the nonthermal velocity as derived from Si VIII 1445.75 $\AA$ (Banerjee et al. 1998). The dashed line is a third-order polynomial fit.

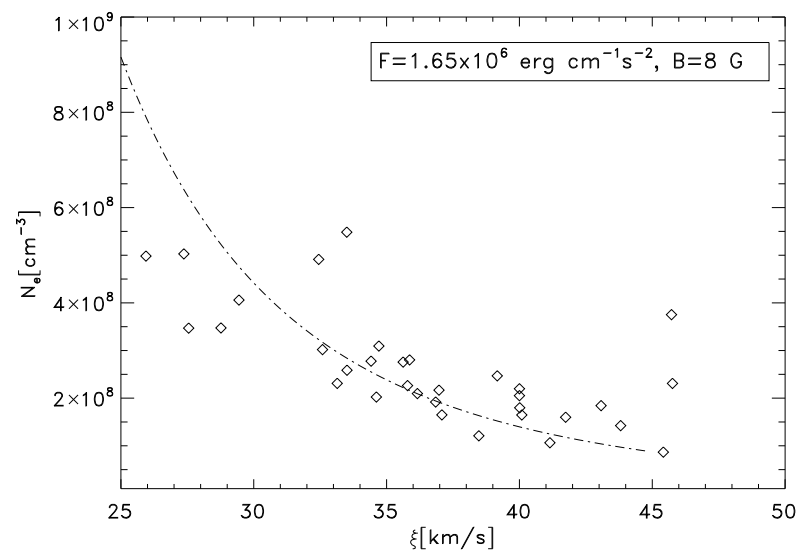

Fig. 5. Variation in electron density with nonthermal velocity for the polar coronal hole. The squared boxes represents the observed values and the solid line represents the theoretical relation (Eq. (4)) for fixed magnetic field strength as indicated.

This suggests that the coronal emission may have been affected by background emission from the quiet coronal structures.

Alfvén waves propagate virtually undamped through the quasi-static corona and deposit their energy flux in the higher corona. The total energy flux crossing a surface of area $A$ in the corona due to Alfvén waves is given by (Moran 2001)

$F=\sqrt{\frac{\rho}{4 \pi}}\left\langle\delta v^{2}\right\rangle B A$,

where $\rho$ is the plasma mass density (related to $N_{\mathrm{e}}$ as $\rho=m_{\mathrm{p}} N_{\mathrm{e}}$, $m_{\mathrm{p}}$ is the proton-mass), $\left\langle\delta v^{2}\right\rangle$ is the mean square velocity related to the observed nonthermal velocity as $\xi^{2}=\left\langle\delta v^{2}\right\rangle / 2$, and $B$, is the magnetic field strength. If the wave energy flux is conserved as the wave propagate outwards, then Eq. (2) gives

$\left\langle\delta v^{2}\right\rangle^{1 / 2} \propto \rho^{-1 / 4}(B A)^{-1 / 2}$.

Now if one assumes a flux tube geometry, then $B A$ is constant with height and Eq. (3) yields

$\left\langle\delta v^{2}\right\rangle^{1 / 2} \propto \rho^{-1 / 4}$.
From our dataset (for Fe XII $195 \AA$ ) at $30^{\prime \prime}$ above the limb for the interplume location (see Table 1) using $N_{\mathrm{e}}=2.2 \times 10^{8} \mathrm{~cm}^{-3}$, $\left\langle\delta v^{2}\right\rangle=2 \times\left(43.7 \mathrm{~km} \mathrm{~s}^{-1}\right)^{2}$, we find $F=1.85 \times 10^{6} \mathrm{erg} \mathrm{cm}^{-2} \mathrm{~s}^{-1}$ for $B=8 \mathrm{G}$, which is only slightly higher than the requirements for a coronal hole with a high-speed solar wind flow (Withbroe \& Noyes 1977), which is in turn estimated to be $8 \times$ $10^{5} \mathrm{erg} \mathrm{cm}^{-2} \mathrm{~s}^{-1}$. The average field strength in coronal holes is estimated to be 5-10 G (Hollweg 1990; Tsuneta et al. 2008a).

To explore the validity of Eq. (4), we plot Fig. 5 corresponding to our interplume data. The solid lines are the theoretically predicted functional form of the variation of number density with nonthermal velocity (Eq. (4)) and the diamonds are our observed data. The proportionality constant have been chosen to match the calculated energy flux. For the interplume data we used $B=8 \mathrm{G}$. Once again the agreement is very good, especially when we are away from the limb.

Our observations provide observational signatures for the presence of Alfvén waves in polar coronal regions at least within $1.1 R_{\odot}$. These upwardly propagating Alfvén waves may heat the corona and accelerate the solar wind. The slightly larger widths within the interplume regions as compared to plumes also indicate that probably interplumes are the preferred channel for the acceleration of the wind. In this dataset we do not find large differences between plumes and interplumes beyond 70" above the limb. Finally the EIS line width results are consistent with previous results from SUMER.

Acknowledgements. Research at Armagh Observatory is grant-aided by the N. Ireland Dept. of Culture, Arts, and Leisure. This work was supported by a Royal Society/British Council and STFC grant PP/D001129/1. The SUMER project is financially supported by DLR, CNES, NASA, and the ESA PRODEX programme (Swiss contribution). We thank the referee for valuable suggestions, which has improved the quality of this article.

\section{References}

Antonucci, E., Giordano, S., Benna, C., et al. 1997, in Fifth SOHO Workshop, The Corona and Solar Wind Near Minimum Activity, ed. A. Wilson, ESA SP, 404, 175

Banerjee, D., Teriaca, L., Doyle, J. G., \& Wilhelm, K. 1998, A\&A, 339, 208 Banerjee, D., Teriaca, L., Doyle, J. G., \& Lemaire, P. 2000, Sol. Phys., 194, 43 Culhane, J. L., Harra, L. K., James, A. M., et al. 2007, Sol. Phys., 243, 19 De Pontieu, B., McIntosh, S., Hansteen, V. H., et al. 2007a, PASJ, 59, 655 De Pontieu, B., McIntosh, S. W., Carlsson, M., et al. 2007b, Science, 318, 1574 Del Zanna, G., \& Mason, H. E. 2005, A\&A, 433, 731

Dolla, L., \& Solomon, J. 2008, A\&A, 483, 271

Doyle, J. G., Banerjee, D., \& Perez, M. E. 1998, Sol. Phys., 181, 91

Giordano, S., Antonucci, E., Noci, G., Romoli, M., \& Kohl, J. L. 2000, ApJ, 531, L79

Hassler, D. M., \& Moran, T. G. 1994, Space Sci. Rev., 70, 373

Hassler, D. M., Rottman, G. J., Shoub, E. C., \& Holzer, T. E. 1990, ApJ, 348, L77

Hassler, D. M., Wilhelm, K., Lemaire, P., \& Schühle, U. 1997, Sol. Phys., 175, 375

Hollweg, J. V. 1990, Comput. Phys. Rep., 12, 205

Kosugi, T., Matsuzaki, K., Sakao, T., et al. 2007, Sol. Phys., 243, 3 Landi, E., Del Zanna, G., Young, P. R., et al. 2006, ApJS, 162, 261 Moran, T. G. 2001, A\&A, 374, L9

Noci, G., Kohl, J. L., Antonucci, E., et al. 1997, Adv. Space Res., 20, 2219 Teriaca, L., Poletto, G., Romoli, M., \& Biesecker, D. A. 2003, ApJ, 588, 566 Tomczyk, S., McIntosh, S. W., Keil, S. L., et al. 2007, Science, 317, 1192 Tsuneta, S., Ichimoto, K., Katsukawa, Y., et al. 2008a, ApJ, 688, 1374 Tsuneta, S., Ichimoto, K., Katsukawa, Y., et al. 2008b, Sol. Phys., 249, 167 van de Hulst, H. C. 1950, Bull. Astron. Inst. Netherlands, 11, 135 Watanabe, T., Hara, H., Yamamoto, N., et al. 2009, ApJ, 692, 1294 Wilhelm, K., Curdt, W., Marsch, E., et al. 1995, Sol. Phys., 162, 189 Wilhelm, K., Marsch, E., Dwivedi, B. N., et al. 1998, ApJ, 500, 1023 Wilhelm, K., Dwivedi, B. N., \& Teriaca, L. 2004, A\&A, 415, 1133 Wilhelm, K., Fludra, A., Teriaca, L., et al. 2005, A\&A, 435, 733 Withbroe, G. L., \& Noyes, R. W. 1977, ARA\&A, 15, 363 Young, P. R., Watanabe, T., Hara, H., \& Mariska, J. T. 2009, A\&A, 495, 587 
D. Banerjee et al.: Alfvén waves in the polar coronal holes, Online Material p 1

Table 1. A summary of measured intensity, calculated nonthermal velocity $(\xi)$, flux ratio and electron density for the plume (P) and interplume (I-P) obtained from Fe XII $\left(T_{i}=1.3 \times 10^{6} \mathrm{~K}\right)$ and Fe XIII $\left(T_{i}=1.6 \times 10^{6} \mathrm{~K}\right)$ at different locations.

\begin{tabular}{|c|c|c|c|c|c|c|c|c|c|c|c|c|c|c|c|c|}
\hline \multirow[t]{2}{*}{$\begin{array}{l}\text { Above limb } \\
\text { (arcsec) }\end{array}$} & \multicolumn{2}{|c|}{$\begin{array}{c}I(195.12 \AA) \\
\mathrm{erg} \mathrm{cm}^{2} \mathrm{~s}^{-1} \mathrm{sr}^{-1}\end{array}$} & \multicolumn{2}{|c|}{$\begin{array}{c}\xi \\
\mathrm{km} \mathrm{s}^{-1}\end{array}$} & \multicolumn{2}{|c|}{$\begin{array}{c}\text { Fe XII ratio } \\
10^{-2}\end{array}$} & \multicolumn{2}{|c|}{$\log N_{\mathrm{e}} / \mathrm{cm}^{-3}$} & \multicolumn{2}{|c|}{$\begin{array}{c}I(202.04 \AA) \\
\operatorname{erg~cm}^{2} \mathrm{~s}^{-1} \mathrm{sr}^{-1}\end{array}$} & \multicolumn{2}{|c|}{$\begin{array}{c}\xi \\
\mathrm{km} \mathrm{s}^{-1}\end{array}$} & \multicolumn{2}{|c|}{$\begin{array}{c}\text { Fe XIII ratio } \\
10^{-2}\end{array}$} & \multicolumn{2}{|c|}{$\overline{l o g} N_{\mathrm{e}} / \mathrm{cm}^{-3}$} \\
\hline & I-P & $\mathrm{P}$ & I-P & $P$ & I-P & $\mathrm{P}$ & I-P & $\mathrm{P}$ & I-P & $\mathrm{P}$ & I-P & $\mathrm{P}$ & I-P & $\mathrm{P}$ & I-P & $\mathrm{P}$ \\
\hline 0.00 & 52.45 & 164.67 & 34.6 & 36.9 & 20.25 & 22.71 & 8.84 & 8.95 & 34.35 & 93.85 & 20.7 & 24.9 & 50.52 & 64.70 & 9.35 & 9.53 \\
\hline 1.00 & 62.02 & 235.34 & 29.3 & 38.4 & 19.27 & 24.54 & 8.80 & 9.03 & 41.81 & 137.62 & 26.1 & 27.9 & 38.72 & 84.76 & 9.19 & 9.78 \\
\hline 2.00 & 69.19 & 338.49 & 28.3 & 39.6 & 21.29 & 25.57 & 8.89 & 9.07 & 48.19 & 190.09 & 22.2 & 27.6 & 22.87 & 86.29 & 8.92 & 9.80 \\
\hline 3.00 & 75.52 & 466.16 & 29.7 & 35.1 & 17.08 & 26.58 & 8.69 & 9.11 & 52.97 & 249.19 & 29.2 & 28.1 & 17.85 & 87.73 & 8.80 & 9.82 \\
\hline 4.00 & 78.00 & 591.03 & 26.7 & 33.5 & 15.42 & 27.77 & 8.61 & 9.15 & 57.13 & 304.34 & 25.9 & 28.0 & 19.98 & 88.91 & 8.85 & 9.84 \\
\hline 5.00 & 77.34 & 675.66 & 24.7 & 29.2 & 14.12 & 29.85 & 8.54 & 9.23 & 60.63 & 341.56 & 31.7 & 28.8 & 15.58 & 87.72 & 8.73 & 9.82 \\
\hline 6.00 & 77.36 & 723.83 & 24.5 & 27.3 & 17.29 & 31.22 & 8.70 & 9.28 & 64.50 & 361.62 & 35.6 & 28.6 & 10.73 & 86.09 & 8.57 & 9.80 \\
\hline 7.00 & 79.45 & 706.95 & 26.0 & 25.5 & 14.13 & 32.34 & 8.54 & 9.32 & 65.34 & 363.00 & 29.4 & 25.5 & 23.97 & 82.00 & 8.94 & 9.75 \\
\hline 8.00 & 79.13 & 664.77 & 23.0 & 25.2 & 17.20 & 32.43 & 8.70 & 9.32 & 65.01 & 347.64 & 29.3 & 24.1 & 15.04 & 77.39 & 8.72 & 9.68 \\
\hline 9.00 & 81.20 & 592.35 & 30.8 & 25.1 & 18.07 & 31.95 & 8.74 & 9.31 & 66.42 & 318.12 & 28.2 & 24.1 & 6.45 & 71.80 & 8.34 & 9.61 \\
\hline 10.00 & 80.88 & 505.33 & 32.0 & 24.1 & 13.21 & 31.79 & 8.49 & 9.30 & 67.35 & 277.62 & 29.7 & 25.3 & 16.50 & 63.80 & .76 & 9.52 \\
\hline 12.00 & 80.63 & 339.73 & 29.9 & 22.1 & 13.02 & 29 & 8.48 & 9.22 & 66.44 & 183.97 & 29.8 & 25.9 & 7.67 & 42.09 & 41 & 9.24 \\
\hline 1 & 78.40 & 201.39 & 30.5 & 28.4 & 11.05 & 10 & 8.36 & 8.82 & 65.57 & 125.56 & 33.0 & 3.2 & 12.94 & 23 & 65 & 8.96 \\
\hline 18 & .91 & 165.77 & 30.8 & 30.4 & 11.85 & 15 & 8.41 & 8. & 67.02 & 113.20 & 30.8 & 26.6 & 14.58 & 22.32 & 70 & 8.90 \\
\hline 21.00 & 71.94 & 144.86 & 34.3 & 29.4 & 10.61 & 12. & 8.34 & 8.46 & 61.95 & 103.00 & 31.1 & 27.9 & 21.06 & 13.27 & 8 & 8.66 \\
\hline 24.00 & 68.51 & 131.47 & 33.0 & 27.3 & 12.33 & 13.57 & 8.44 & 8.51 & 60.72 & 97.82 & 31.6 & 28.2 & 18.04 & 10.72 & 0 & 8.57 \\
\hline 27.00 & 66.44 & 121.21 & 33.1 & 28.4 & 10.90 & 11.48 & 8.35 & 8.39 & 59.08 & 95.80 & 33.7 & 30.7 & 6.55 & 12.26 & 8.34 & 8.63 \\
\hline 30.00 & 63.69 & 116.30 & 34.2 & 27.0 & 9.80 & 10.66 & 8.28 & 8.34 & 56.08 & 89.60 & 31.6 & 31.2 & 0.70 & 16.14 & 7.50 & 8.75 \\
\hline 36.00 & 57.09 & 100.84 & 31.9 & 29.7 & 10.15 & 11.06 & 8.31 & 8.36 & 52.96 & 81.91 & 32.2 & 29.4 & 8.66 & 11.76 & 8.47 & 8.61 \\
\hline 41.00 & 53.43 & 93.63 & 31.7 & 27.3 & 12.38 & 9.2 & 8.44 & 8.24 & 50.69 & 76.62 & 35.4 & 30.1 & 12.21 & 13.79 & 8.62 & 8.68 \\
\hline 46.00 & 52.43 & 86.73 & 35.8 & 28.1 & 7.16 & 9.1 & 8.08 & 8.24 & 48.36 & 71.34 & 35.6 & 32.9 & 5.60 & 7.19 & 8.27 & 8.39 \\
\hline 51.00 & 48.57 & 81.15 & 33.5 & 33.0 & 10.38 & 10.60 & 8.32 & 8.34 & 46.97 & 70.03 & 35.1 & 32.0 & 5.76 & 8.00 & 8.29 & 8.43 \\
\hline 56.00 & 46.51 & 74.57 & 36.5 & 32.6 & 11.52 & 9.6 & 8.39 & 8.27 & 43.59 & 61.55 & 35.0 & 31.9 & 16.10 & 13.13 & 8.75 & 8.66 \\
\hline 00 & 42.88 & 65.98 & 34.4 & 31.4 & 8.86 & 11.49 & 8.22 & 8.39 & 40.57 & 57.00 & 36.3 & 31.5 & 6.72 & 8.19 & 8.36 & 8.44 \\
\hline 66.00 & 39.34 & 61.77 & 37.4 & 32.6 & 10.24 & 10.56 & 8.31 & 8.33 & 39.13 & 52.93 & 34.3 & 32.6 & 4.94 & 11.07 & 8.22 & 8.58 \\
\hline 71.00 & 40.04 & 57.45 & 40.4 & 33.3 & 9.55 & 8.65 & 8.27 & 8.20 & 36.33 & 50.11 & 35.7 & 32.6 & 2.08 & 8.64 & 7.82 & 8.47 \\
\hline 77.00 & 36.04 & 52.79 & 37.4 & 34.8 & 10.70 & 9.90 & 8.34 & 8.29 & 34.49 & 46.50 & 39.7 & 35.4 & 14.54 & 19.55 & 8.70 & 8.84 \\
\hline 84.00 & 31.71 & 46.21 & 37.4 & 32.6 & 8.86 & 9.2 & 8.22 & 8.24 & 29.95 & 41.55 & 40.9 & 35.8 & 10.53 & 10.27 & 8.56 & 8.55 \\
\hline 91.00 & 29.47 & 41.63 & 38.5 & 35.8 & 6.55 & 6.9 & 8.03 & 8.06 & 26.83 & 36.19 & 38.6 & 35.8 & 4.22 & 14.17 & 8.15 & 8.69 \\
\hline 98.00 & 26.06 & 36.87 & 39.1 & 35.6 & 8.68 & 9.0 & 8.20 & 8.23 & 24.20 & 32.70 & 44.6 & 40.2 & 5.23 & 9.89 & 8.24 & 8.53 \\
\hline 105.00 & 24.47 & 34.22 & 43.1 & 34.1 & 11.04 & 10.78 & 8.36 & 8.35 & 22.11 & 29.07 & 35.3 & 32.3 & 6.19 & 9.02 & 8.32 & 8.49 \\
\hline 2.00 & 23.00 & 31.14 & 37.4 & 34.6 & 9.39 & 6.49 & 8.25 & 8.02 & 21.09 & 29.55 & 41.2 & 38.9 & 14.96 & -2.76 & 8.72 & $* * * *$ \\
\hline 122.50 & 20.49 & 28.04 & 43.0 & 39.0 & 14.76 & 9.8 & 8.57 & 8.29 & 17.72 & 24.00 & 40.2 & 37.4 & 6.88 & 5.61 & 8.37 & 8.27 \\
\hline 136.50 & 16.80 & 22.50 & 41.1 & 39.8 & 8.02 & 5.2 & 8.15 & 7.89 & 14.61 & 19.07 & 37.5 & 37.4 & 5.24 & 12.69 & 8.24 & 8.64 \\
\hline 150.50 & 14.68 & 18.72 & 42.7 & 40.3 & 5.66 & 8.82 & 7.94 & 8.21 & 12.31 & 15.80 & 44.0 & 39.5 & 0.05 & 6.85 & 7.50 & 8.36 \\
\hline
\end{tabular}
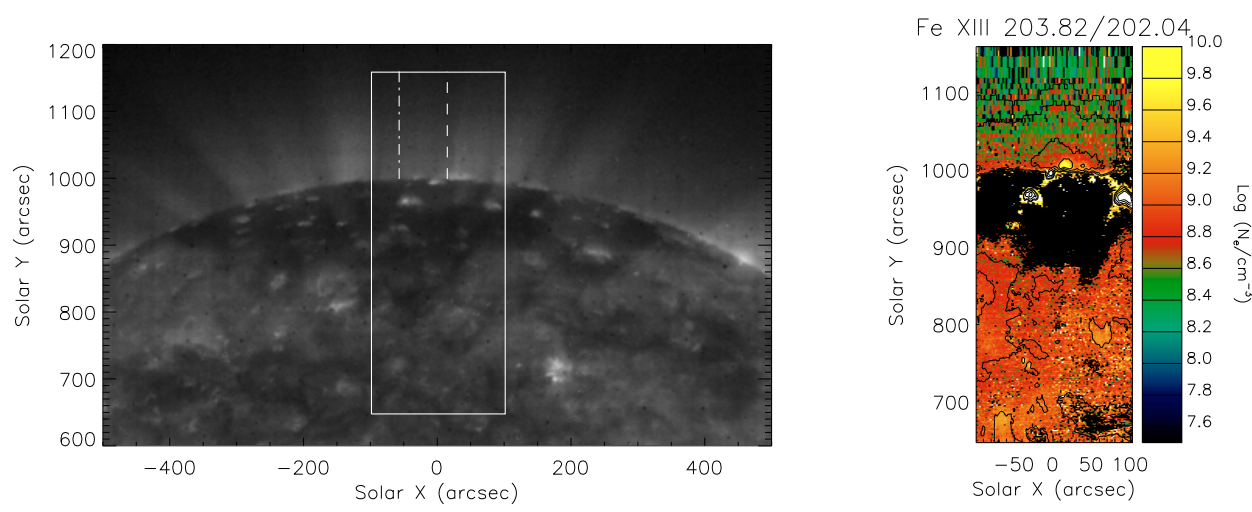

Fig. 6. The left panel shows the EIT $171 \AA$ image with EIS field of view overplotted (square box) and plume and interplume chosen. The right panel shows the density map corresponding to the full EIS raster scan as recorded by Fe XIII and the contours show the intensity. 